\title{
Column-scale unsaturated hydraulic conductivity estimates in coarse-textured homogeneous and layered soils derived under steady-state evaporation from a water table
}

\author{
Morteza Sadeghi ${ }^{\mathrm{a}, *}$, Markus Tuller ${ }^{\mathrm{b}}$, Mohammad R. Gohardoust ${ }^{\mathrm{c}}$, Scott B. Jones ${ }^{\mathrm{a}}$ \\ ${ }^{a}$ Dept. Plants, Soils and Climate, Utah State University, Logan, UT, USA \\ ${ }^{\mathrm{b}}$ Dept. Soil, Water, and Environmental Science, The University of Arizona, Tucson, AZ, USA \\ ${ }^{\mathrm{c}}$ Dept. Water Engineering, Sari Agricultural Sciences and Natural Resources University, Sari, Iran
}

\section{A R T I C L E I N F O}

\section{Article history:}

Received 6 July 2014

Received in revised form 29 August 2014

Accepted 2 September 2014

Available online 10 September 2014

This manuscript was handled by Peter K.

Kitanidis, Editor-in-Chief, with the

assistance of J.A. Huisman, Associate Editor

\section{Keywords:}

Steady-state evaporation

Unsaturated hydraulic conductivity

Heterogeneity

Upscaling

Effective hydraulic properties

\begin{abstract}
S U M M A R Y
Steady-state evaporation from a water table has been extensively studied for both homogeneous and layered porous media. For layered media it is of interest to find an equivalent homogeneous medium and define "effective" hydraulic properties. In this paper a new solution for steady-state evaporation from coarse-textured porous media is presented. Based on this solution, the evaporation rate represents a macroscopic (column-scale) measure of unsaturated hydraulic conductivity at the pressure head equal to the maximum extent of the hydraulically connected region above the water table. The presented approach offers an alternative method for determination of unsaturated hydraulic conductivity of homogeneous coarse-textured soils and a new solution for prediction of the effective unsaturated hydraulic conductivity of layered coarse-textured soils. The solution was evaluated with both experimental data and numerical simulations. Comparison with experimental data and numerical results for hypothetically layered soil profiles demonstrate the applicability of the proposed approach for coarse-textured soils.
\end{abstract}

(C) 2014 Elsevier B.V. All rights reserved.

\section{Introduction}

One-dimensional steady-state evaporation from a fixed shallow water table (WT) is dependent on the WT depth and on the soil hydraulic properties (Lehmann et al., 2008; Shokri and Salvucci, 2011; Assouline et al., 2013; Or et al., 2013). Two distinct scenarios are often considered; the first when the WT is shallow enough for the soil to sustain hydraulic continuity between the WT and the surface due to capillary forces, and the second when the WT reaches a critical depth (denoted as $D_{\max }$ ) below which the hydraulic continuity between the WT and soil surface is no longer maintained.

In the first scenario liquid water continuously flows from the WT to the surface where it transitions to the vapor phase. The maximum WT depth $\left(D_{\max }\right)$ for which hydraulic continuity can

Abbreviations: BC, Brooks and Corey; DF, drying front; TO, Tuller and Or; VG, van Genuchten; WT, water table.

* Corresponding author at: 4820 Old Main Hill, Logan, UT 84322-4820, USA. Tel.: +1 435797 2233; fax: +1 4357973376 .

E-mail addresses: morteza.sadeghi@usu.edu (M. Sadeghi), mtuller@cals.arizona. edu (M. Tuller), gohardoust@gmail.com (M.R. Gohardoust), scott.jones@usu.edu (S.B. Jones). be sustained depends on the porous medium properties and on the evaporation rate, which in this case is mainly controlled by the atmospheric evaporative demand. In the second scenario liquid water flows from the WT to the so-called drying front (DF), where it transitions to the vapor phase and moves to the surface by means of vapor diffusion.

Steady-state water flow from the WT to the soil surface was extensively studied theoretically and experimentally for homogeneous soils (Moore, 1939; Gardner, 1958; Anat, 1965; Warrick, 1988; Gowing et al., 2006; Salvucci, 1993; Shokri and Salvucci, 2011; Sadeghi et al., 2012a) as well as for stratified soil profiles (Willis, 1960; Ripple et al., 1970; Warrick and Yeh, 1990; Lu and Zhang, 2004) and other heterogeneous media (Hopmans et al., 1998; Zhu and Mohanty, 2002; Bechtold et al., 2012). The majority of developed models are based on the Buckingham-Darcy law (Buckingham, 1907) and its analytical or numerical solutions within the liquid flow domain. The analytical solutions are commonly restricted to simple forms of hydraulic conductivity models such as proposed by Gardner (1958) or Brooks and Corey (1964).

When dealing with complex heterogeneous media it is of interest to define "effective" or "upscaled" hydraulic properties for an equivalent homogeneous medium. A vast body of literature exists 
on upscaling issues for various soil water processes including steady-state flow (Yeh and Harvey, 1990; Zhu and Mohanty, 2002; Warrick, 2005; Neuweiler and Eichel, 2006). There are different approaches with regard to scaling of soil hydraulic properties and soil water processes in heterogeneous porous media such as averaging methods (Kitanidis, 1990; Zhu et al., 2004; Zhu and Mohanty, 2006), inverse solutions (van Dam et al., 1994; Hopmans and Simunek, 1999; Zhang et al., 2004; Javaux and Vanclooster, 2006; Vrugt et al., 2008; Erdal et al., 2012), percolation theory (Hunt, 1998; Samouelian et al., 2007; Hunt and Idriss, 2009), homogenization theory (Neuweiler and Cirpka, 2005; Neuweiler and Eichel, 2006; Neuweiler and Vogel, 2007), hybrid mixture theory (Hassanizadeh and Gray, 1979, 1979, 1980), similar media scaling theory (Miller and Miller, 1956; Warrick and Amoozegar-Fard, 1979; Kosugi and Hopmans, 1998; Sadeghi and Jones, 2012; Ojha et al., 2014), dissimilar media scaling methods (Sadeghi et al., 2012b,c), stochastic methods (Yeh et al., 1985; Zhang and Lu, 2002), and renormalization methods (King, 1989; Saucier, 1992; King and Neuweiler, 2002). Many of these methods and their strengths and limitations have been previously reviewed and discussed (e.g., Wen and Gomez-Hernandez, 1996; Pachepsky et al., 2003; Cushman et al., 2002; Chen, 2006; Vereecken et al., 2007).

In this paper a new general solution to the maximum height of hydraulic continuity above the water table $\left(D_{\max }\right)$ in coarsetextured porous media is presented. Here, "general" means that the solution is not restricted to a specific form of the unsaturated hydraulic conductivity function, but applicable to many existing mathematical relationships. The solution provides an alternative method for determination of unsaturated hydraulic conductivity of homogeneous coarse-textured soils and a new solution for effective or upscaled unsaturated hydraulic conductivity of layered coarse-textured media. Various aspects of applicability of the proposed solution to coarse-textured soils are presented and discussed in the following.

\section{Theoretical considerations}

\subsection{Background}

We begin by considering an isothermal steady-state evaporation process during stage II (vapor diffusion limiting stage). This process can be modeled with the Buckingham-Darcy law considering both liquid and vapor flow contributions to the unsaturated hydraulic conductivity (Peters, 2013):

$e=K\left(\frac{d h}{d z}-1\right)=\left(K_{l}+K_{v}\right)\left(\frac{d h}{d z}-1\right)$

where $h$ is the pressure head (absolute value), $z$ is the vertical distance from the WT to the soil surface (positive upward), $e$ is the steady-state evaporation rate, and $K_{l}, K_{v}$, and $K$ are the liquid, vapor, and total hydraulic conductivities, respectively.

Assuming a steady condition, the pressure head distribution above the water table, $h(z)$, can be determined by solving a rearranged form of Eq. (1):

$z=\int_{0}^{h} \frac{K(h)}{K(h)+e} d h$

Fig. 1 depicts a sketch of the pressure head profile above the water table obtained by numerically solving Eq. (2) considering both liquid and vapor flow contributions (i.e., $K=K_{l}+K_{v}$ ) and liquid flow only (i.e., $K=K_{l}$ ). Three different zones are distinguished along the profile: (i) a saturated zone known as the capillary fringe $\left(0<h<h_{b}\right.$, where $h_{b}$ is the air-entry pressure head); (ii) an intermediate, partially-saturated zone between the capillary fringe and the drying front (DF) $\left(h_{b}<h<h_{\max }\right.$, where $h_{\max }$ is the pressure head at

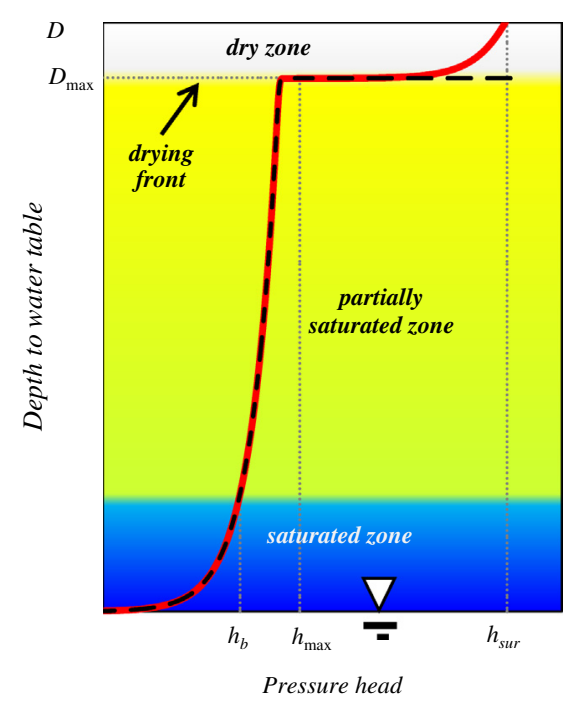

Fig. 1. Sketch depicting the pressure head distribution above the water table during steady-state evaporation with (solid line) and without (dashed line) vapor flow contribution (film flow contribution as shown by Peters and Durner (2010) is not considered here). In this figure, $h_{b}$ denotes the air-entry pressure head, $h_{\max }$ is pressure head at the drying front where liquid continuity is disrupted, $h_{\text {sur }}$ is pressure head at the surface, $D$ is the water table depth, and $D_{\max }$ is the maximum extent of the hydraulically connected region above the water table. Pressure head is plotted on a logarithmic scale.

which hydraulic liquid continuity is disrupted); and (iii) a dry zone (gas region) between the DF and the soil surface $\left(h_{\max }<h<h_{\text {sur }}\right.$, where $h_{\text {sur }}$ is the pressure head at the soil surface). It is evident that the pressure head distribution within the dry zone cannot be modeled without accounting for the vapor flow contribution. However, the solution along the liquid flow domain (i.e., $0<z<D_{\max }$ ) is the same for both cases.

The pressure head at the surface $\left(h_{\text {sur }}\right)$ depends on the atmospheric boundary conditions and can be estimated as (Edelfsen and Anderson, 1943):

$h_{\text {sur }}=\frac{R T\left|\ln H_{r}\right|}{M g}$

where $R\left(=8.314 \mathrm{~J} \mathrm{~mol}^{-1} \mathrm{~kg}^{-1}\right)$ is the ideal gas constant, $T$ is temperature (in Kelvin), $M\left(=0.018015 \mathrm{~kg} \mathrm{~mol}^{-1}\right)$ is the molecular weight of water, $g\left(=9.81 \mathrm{~m} \mathrm{~s}^{-2}\right)$ is gravitational acceleration, and $H_{r}$ is relative humidity.

Based on the numerically simulated pressure head profile shown in Fig. 1 as well as results of Peters and Durner (2010) and Peters (2013), the pressure head at DF $\left(h_{\max }\right)$ is realized as a critical value at which liquid and vapor conductivity curves meet each other (i.e., $\left.K_{l}\left(h_{\max }\right)=K_{v}\left(h_{\max }\right)\right)$. Below $h_{\max }$, liquid water flow dominates transport and above $h_{\max }$ vapor flow is dominant. This definition is similar to that of Assouline et al. (2014) (their Eq. (25)) who described $h_{\max }$ as the pressure head corresponding to the water content marking the onset of stage II (vapor diffusion limiting stage) evaporation.

Dependence of liquid conductivity on pressure head may be described by an empirical relationship (e.g., Brooks and Corey, 1964; van Genuchten, 1980) or a physically-based model (e.g., Tuller and Or, 2001). The vapor conductivity function can be determined from (Philip and De Vries, 1957; Fayer, 2000):

$K_{v}(h)=\frac{\rho_{s v}}{\rho_{w}} D_{v} \frac{M g}{R T} H_{r}=\frac{\rho_{s v}}{\rho_{w}} D_{v} \frac{M g}{R T} \exp \left(-\frac{M g}{R T} h\right)$

where $\rho_{w}$ is liquid water density $\left(=1000 \mathrm{~kg} \mathrm{~m}^{-3}\right), \rho_{v s}$ is the saturated vapor density $\left(=0.017 \mathrm{~kg} \mathrm{~m}^{-3}\right.$ at $\left.20^{\circ} \mathrm{C}\right)$, and $D_{v}$ is the soil vapor diffusivity given as: 
$D_{v}=\tau \theta_{a} D_{a}=\frac{\theta_{a}^{7 / 3}}{\theta_{s}^{2}} \theta_{a} D_{a}$

where $\tau$ is the tortuosity factor described by Millington and Quirk (1961), $\theta_{a}$ is the volumetric air content, $D_{a}$ is the diffusivity of water vapor in air $\left(=2.44 E-5 \mathrm{~m}^{2} \mathrm{~s}^{-1}\right)$, and $\theta_{s}$ is the saturated soil water content.

Once water table depth, $D$, the pressure head at the soil surface, $h_{\text {sur }}$, and the soil liquid and vapor hydraulic conductivity functions, $K(h)$, are known, we can implicitly calculate the steady-state evaporation rate during stage II based on Eq. (2), rewritten as:

$D=\int_{0}^{h_{\text {sur }}} \frac{K(h)}{K(h)+e} d h$

Then, $D_{\max }$ can be determined as:

$D_{\max }=\int_{0}^{h_{\max }} \frac{K(h)}{K(h)+e} d h$

\subsection{New solution for $D_{\max }$}

Eq. (7) may be rearranged to read:

$D_{\max }=\int_{0}^{h_{\max }} \frac{K(h)+e-e}{K(h)+e} d h=h_{\max }-e \int_{0}^{h_{\max }} \frac{d h}{K(h)+e}$

The function $1 /[K(h)+e]$ in Eq. (8) typically exhibits an "S" shape as shown in Fig. 2. For most coarse-textured soils where the $K(h)$ function exhibits a steep slope and the saturated hydraulic conductivity $\left(K_{s}\right)$ is much larger than common values of $e$, the areas $A_{1}$ and $A_{2}$ in Fig. 2 tend to be identical. Hence, Eq. (8) for such conditions can be approximated as:

$D_{\max }=h_{\max }-e\left[\int_{0}^{h_{e}} \frac{d h}{K_{s}}+\int_{h_{e}}^{h_{\max }} \frac{d h}{e}\right]=h_{e}\left(1-\frac{e}{K_{s}}\right)$

where $h_{e}$ is the pressure head at which $K=e$. Because Eq. (9) holds for coarse-textured soils with $e / K_{\mathrm{s}}$ being negligible, the solution can be further simplified to:

$D_{\max }=h_{e}$

Eq. (10) states that for coarse-textured porous media, pairs of $e-D_{\max }$ values coincide with the unsaturated hydraulic conductivity curve, $K(h)$, which means that the steady-state evaporation rate exhibits a measure for unsaturated hydraulic conductivity at the pressure head equal to $D_{\max }$. In summary, when $h=D_{\max }, K=e$.

The universal formulation of $D_{\max }$ presented above may provide an additional opportunity for modeling evaporation by offering new solutions to the drying front depth, $\delta$, which has been applied

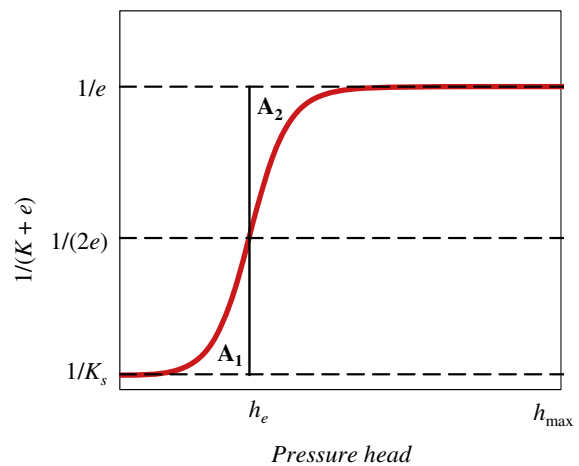

Fig. 2. Mathematical behavior of the $1 /[K(h)+e]$ function in Eq. (8). as a key part of the stage II evaporation modeling (Or et al., 2013). Some connections between $D_{\max }, \delta$, and $e$ are thus presented.

Combining Eqs. (6) and (7) yields:

$\delta=D-D_{\max }=\int_{h_{\max }}^{h_{\text {sur }}} \frac{K(h)}{K(h)+e} d h$

Within the dry zone, $K=K_{v}$, which is nearly invariant with respect to pressure head from $h_{\max }$ to $h_{\text {sur }}\left(\approx 1 \mathrm{E} 6 \mathrm{~cm}\right.$ at $20^{\circ} \mathrm{C}$ and $50 \%$ relative humidity) (see Fig. 4). Thus Eq. (11) can be solved and rewritten as:

$\delta=\frac{0.5\left[K_{v}\left(h_{\max }\right)+K_{v}\left(h_{\text {sur }}\right)\right]}{0.5\left[K_{v}\left(h_{\max }\right)+K_{v}\left(h_{\text {sur }}\right)\right]+e}\left(h_{\text {sur }}-h_{\max }\right)$

Assuming $h_{\text {sur }} \gg h_{\max }$ and $e \gg K_{v}$, Eq. (12) yields:

$\delta=\frac{0.5\left[K_{v}\left(h_{\max }\right)+K_{v}\left(h_{\text {sur }}\right)\right] h_{\text {sur }}}{e}=\frac{C}{e}$

where $C$ is a constant under steady-state conditions. Since the Buckingham-Darcy law can be converted to a diffusion-type equation by assuming $D_{v}=K_{v} \mathrm{~d} h / \mathrm{d} \rho$, where $\rho$ is vapor density (Bittelli et al., 2008), Eq. (13) is similar to the previously developed $\delta-e$ relationships based on Fick's law (Gardner, 1958; Shokri et al., 2008, 2009).

Finally, the following analytical solution to Eq. (6) is obtained by combining Eqs. (10) and (13), which can be implicitly solved for evaporation rate as a function of water table depth:

$D=h_{e}+\frac{C}{e}$

Eq. (14) describes how deepening of the water table decreases the evaporation rate necessary for extension of both liquid (first term) and vapor (second term) regions.

\section{Validation and application}

\subsection{Comparison with an analytical solution}

A commonly applied relationship for liquid unsaturated hydraulic conductivity is that of Brooks and Corey (BC) (1964):

$K_{l}= \begin{cases}K_{s} & \left(h \leqslant h_{b}\right) \\ K_{s}\left(h / h_{b}\right)^{-P} & \left(h>h_{b}\right)\end{cases}$

where $h_{b}$ and $P$ are BC model parameters. Applying the BC $K_{l}(h)$ function, Sadeghi et al. (2012a) solved Eq. (7) analytically to obtain a relationship between $D_{\max }, e$, and soil properties:

$$
\begin{aligned}
D_{\max }= & h_{b}\left\{\frac{\ln \left(1+e / K_{s}\right)}{1+P}-\frac{e}{K_{s}+e}\right. \\
& \left.-\left(e / K_{s}\right)^{-1 / P}\left[\frac{2 \ln 2}{1-P^{2}}+\frac{\pi^{2} / 12-\ln 2}{P(1-P)}-1\right]\right\}
\end{aligned}
$$

Assuming a steep slope of the $K_{l}(h)$ function (i.e. $P$ is large) and that $e / K_{s}$ is negligibly small, Eq. (16) may be approximated as:

$D_{\max }=h_{b}\left(e / K_{s}\right)^{-1 / P}=h_{e}$

This corresponds to Eq. (10) which has been obtained universally for any mathematical relationship of $K_{l}(h)$ which satisfies the two abovementioned assumptions $\left(K_{l}(h)\right.$ is steep and $e / K_{s}$ is negligible). Within this context, this analytical solution was applied to preliminarily define a "coarse-textured" soil.

As an illustrative example, Fig. 3 presents a comparison between the analytical solution (Eq. (16)) and the BC model for a coarse-, a medium-, and a fine-textured soil. Fig. 3 indicates that Eq. (10) is an accurate approximation of Eq. (16) for a coarsetextured soil except near saturation, but underestimates the solution for a fine-textured porous medium. Errors near saturation for 


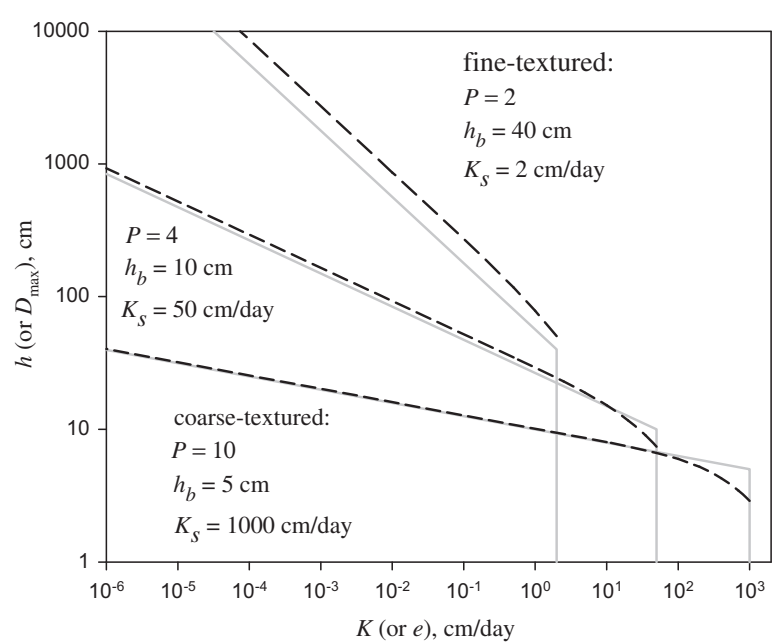

Fig. 3. Comparison between the Brooks-Corey $K_{l}(h)$ function (solid line) and the analytical solution based on the Buckingham-Darcy law (Eq. (16)) (dashed line) for coarse-, medium-,and fine-textured soils.

the coarse-textured soil are because both assumptions for obtaining Eq. (10) are not fulfilled; i.e. $e / K_{s}$ is not negligible and the slope of the $K(h)$ function is not steep. Based on these results, we may roughly say that the "steep slope" (i.e., log-log) can be defined as $P$ greater than 3 or 4 . Also as Fig. 3 and results shown in the following indicate, $e / K_{s}$ can be assumed "negligible" when $e$ is at least one order of magnitude smaller than $K_{s}$ in finer soils or two orders of magnitude smaller in coarser soils.

\subsection{Comparison with numerical solutions}

As discussed above, the solution to Eq. (7) for $D_{\max }$, can be analytically derived only for simple algebraic $K_{l}(h)$ relationships such as the BC power model (Warrick, 1988; Sadeghi et al., 2012a). Lehmann et al. (2008) and Shokri and Salvucci (2011) incorporated the van Genuchten (1980) retention model and derived an approximate analytical solution for $D_{\max }$ of coarse-textured soils by balancing capillary and gravitational forces. But in general, there are numerous mathematically more complex expressions for $K_{l}(h)$ for which an analytical solution for Eq. (2) cannot be easily derived (e.g., Kosugi, 1996; Tuller and Or, 2001).

As shown and discussed above, Eq. (10) may be considered as a general analytical solution for $D_{\max }$ for coarse-textured soils, regardless of the $K_{l}(h)$ mathematical relationship. In the following, we evaluate the validity of Eq. (10) for the van Genuchten (1980), Tuller and $\operatorname{Or}(2001)$, and a bimodal $K_{l}(h)$ model.

Eq. (7) was solved numerically assuming various values of $e$ within the liquid flow domain. As Fig. 1 indicates, since liquid flow is dominant along the $D_{\max }$ domain (i.e., $0<z<D_{\max }$ ), there is no difference in the $D_{\max }$ solution with or without consideration of the vapor flow contribution. Hence, $K$ in Eq. (7) was considered solely as liquid conductivity. In this case, $D_{\max }$ approaches a constant value when the upper limit of the integral in Eq. (7) becomes larger than a threshold and the solution will remain unchanged for pressure head values larger than $h_{\max }$ (see the dashed line in Fig. 1). That is why $h_{\max }$ is not incorporated in the final solution of $D_{\max }$ (e.g., in Eqs. (10) or (16)). Hence, for the numerical solution of Eq. (7), a sufficiently large pressure head was assumed (i.e., yielding the final value of $D_{\max }$ ) as the upper limit of the integral. Hence, this assumption introduced for simplifying calculations only should not be confused with the incorrect assumption of liquid continuity along the entire soil profile discussed by Shokri and $\operatorname{Or}$ (2010). Note that when $K$ in Eq. (7) is considered as the total hydraulic conductivity, the integration should be restricted to the true value of $h_{\max }$, otherwise the calculated $D_{\max }$ will be overestimated.

\subsubsection{The van Genuchten (1980) $K_{l}(h)$ model}

The van Genuchten (VG) (1980) closed-form expression for the unsaturated hydraulic conductivity function based on the Mualem (1976) model is one of the most widely applied $K_{l}(h)$ relationships, given as:

$K_{l}=K_{s} \frac{\left\{1-(\alpha h)^{n-1}\left[1+(\alpha h)^{n}\right]^{-m}\right\}^{2}}{\left[1+(\alpha h)^{n}\right]^{m / 2}}$

where $\alpha, n$, and $m$ are empirical model parameters with the assumption that $m=1-1 / n$.

In order to validate the applicability of Eq. (10), we employed data and hydraulic parameters from the Loveland sand in Anat (1965), the sand studied by Smits et al. (2012) (Sand 1), the sand studied by Prevedello et al. (2009) (Sand 2), and three coarsetextured soils from van Genuchten (1980) including Hygiene sandstone, Touchet silt loam, and Silt loam G.E.3 with VG parameters listed in Table 1.

Before evaluating the proposed solution for $D_{\max }$, we explored the range of pressure head and conductivity values within which water flows predominantly in the liquid state. As mentioned earlier, $h_{\max }$ can be determined by comparing liquid and vapor conductivity curves as shown in Fig. 4 for Sand 1 and Silt loam G.E.3. Soil water retention functions are also presented to see how the liquid flow domain can be defined in terms of soil water content. It is clear that $h_{\max }$ is quite different for various soils (see Table 2 for all soils). However, $K\left(h_{\max }\right)$ for these soils lies within a narrow range, which is roughly between $1 \mathrm{E}-7$ and $1 \mathrm{E}-8 \mathrm{~cm} /$ day. Based on these results and to limit our analysis to the liquid phase, we restricted all of our evaluations to conductivity values greater than $1 \mathrm{E}-7 \mathrm{~cm} /$ day.

Fig. 4 also indicates that, in terms of soil water content, hydraulic continuity can be assumed down to the point where the soil dries to near-residual water content values, which is consistent with the alternative definition of $h_{\max }$ introduced by Lehmann et al. (2008). However, as Fig. 4 shows and similarly reported by Assouline et al. (2014), the Lehmann et al. (2008) approximation $\left(h_{\max } \approx \alpha^{-1}((n-1) / n)^{(1-2 n) / n}\right)$ obtained by linearization of the soil-water retention curve underestimates $h_{\max }$ especially for finer soils.

Numerical solutions of Eq. (7) for the VG $K_{l}(h)$ function (Eq. (18)) in comparison with the VG $K_{l}(h)$ function itself are presented in Fig. 5. The reasonable agreement between the VG $K(h)$ model and numerical results of Eq. (7) indicate that Eq. (10) is valid for the VG function. Some slight disagreements between the numerical and approximate solutions are apparent for Silt loam G.E.3. As mentioned earlier, the solution is expected to work well for coarse-textured soils with an abruptly decreasing $K(h)$ function. Therefore, the small errors for Silt loam G.E.3 are due to its "relatively" low $n$ parameter. As the finest textured soil considered, results for Silt loam G.E.3 indicate that $n(\approx 2)$ may be considered as a lower limit of the new solution's applicability for VG soils (i.e. the solution works well for soils with $n>2$ ).

A slight discrepancy is also seen for Hygiene sandstone at very high evaporation rates. This error is expected due to the "relatively" low $K_{s}$ of this soil and the fact that $e / K_{s}-$ which was assumed to be negligible for obtaining the new solution - becomes significant when $e$ is high.

\subsubsection{The Tuller and Or (2001) $K_{l}(h)$ model}

Classical models like that of Mualem (1976) often rely on oversimplified representation of medium pore space as a bundle of 
Table 1

van Genuchten parameters for considered soils.

\begin{tabular}{|c|c|c|c|c|c|}
\hline Soil name & $K_{s}(\mathrm{~cm} /$ day $)$ & $\alpha\left(\mathrm{cm}^{-1}\right)$ & $n$ & $\theta_{s}$ & $\theta_{r}$ \\
\hline Sand 1 (Smits et al., 2012) & 9158 & 0.0570 & 17.80 & 0.334 & 0.028 \\
\hline Sand 2 (Prevedello et al., 2009) & 1368 & 0.0410 & 17.00 & 0.387 & 0.019 \\
\hline Loveland sand (Anat, 1965) ${ }^{\mathrm{a}}$ & 945 & 0.0490 & 9.79 & 0.430 & 0.045 \\
\hline Hygiene sandstone (van Genuchten, 1980) & 108 & 0.0079 & 10.40 & 0.250 & 0.153 \\
\hline Touchet silt loam (van Genuchten, 1980) & 303 & 0.0050 & 7.09 & 0.469 & 0.190 \\
\hline Silt loam G.E.3 (van Genuchten, 1980) & 5 & 0.0042 & 2.06 & 0.396 & 0.131 \\
\hline
\end{tabular}

a Since $\theta_{s}$ and $\theta_{r}$ were not reported for this soil, common values for a typical sandy soil were assumed.
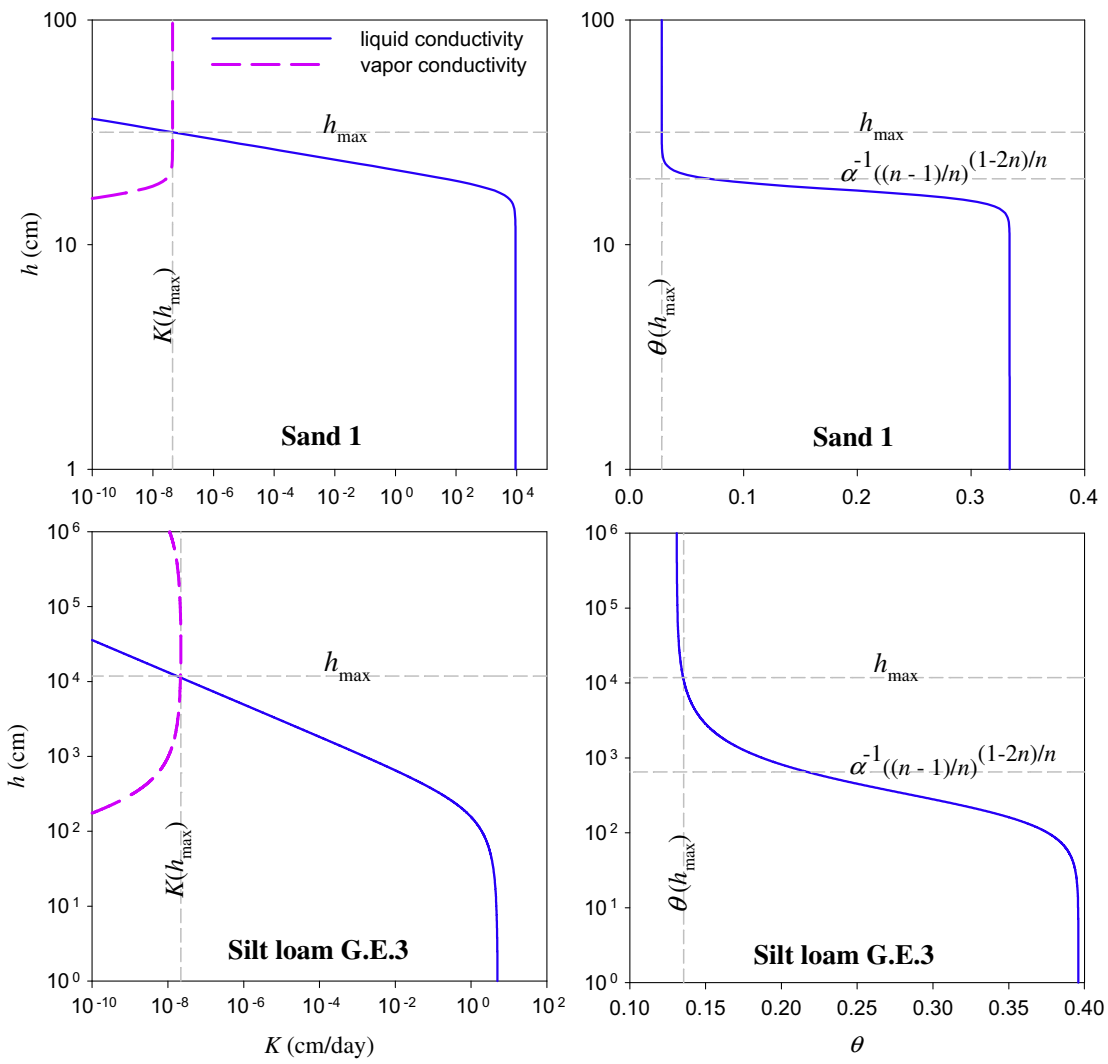

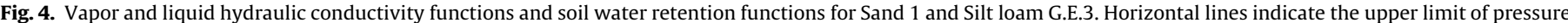

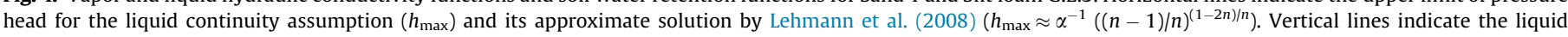
hydraulic conductivity and soil water content at $h=h_{\text {max }}$.

Table 2

Upper limit of pressure head for the liquid continuity assumption $\left(h_{\max }\right)$ and its corresponding liquid hydraulic conductivity and soil water content for the soils considered.

\begin{tabular}{lrll}
\hline Soil name & $h_{\max }(\mathrm{cm})$ & $K_{l}\left(h_{\max }\right)(\mathrm{cm} /$ day $)$ & $\theta\left(h_{\max }\right)$ \\
\hline Sand 1 & 31.63 & $4.49 \mathrm{E}-8$ & 0.028 \\
Sand 2 & 42.66 & $7.70 \mathrm{E}-8$ & 0.019 \\
Loveland sand & 53.70 & $6.42 \mathrm{E}-8$ & 0.045 \\
Hygiene sandstone & 331.13 & $1.98 \mathrm{E}-9$ & 0.153 \\
Touchet silt loam & 776.25 & $1.60 \mathrm{E}-8$ & 0.190 \\
Silt loam G.E.3 & 11220.00 & $2.19 \mathrm{E}-8$ & 0.135 \\
\hline
\end{tabular}

cylindrical capillaries and do not correctly account for thermodynamic pore scale processes assuming that the matric potential is attributed to capillary forces only (ignoring adsorptive surface forces) and flow only occurs in completely full capillaries (Tuller et al., 1999; Or and Tuller, 1999). Tuller and Or (2001) presented a more realistic approach that accounts for film flow contributions in an angular pore space model.

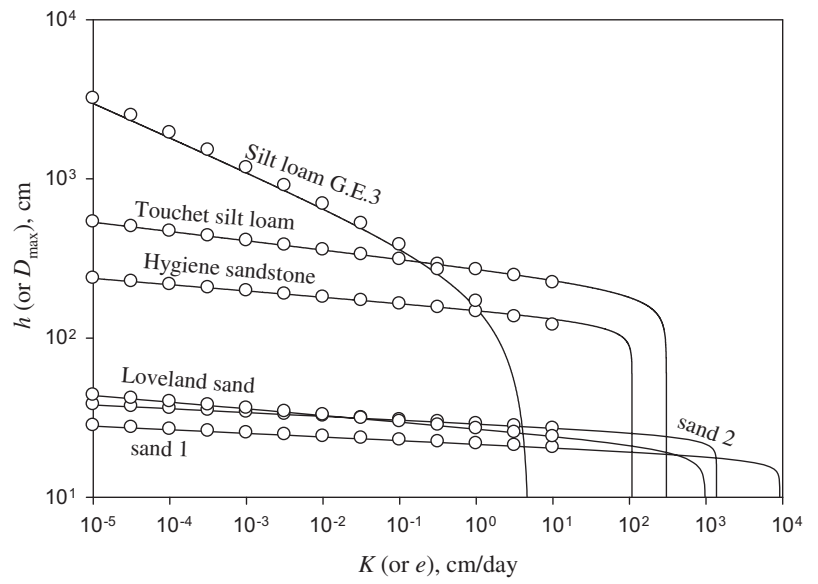

Fig. 5. The van Genuchten (1980) $K_{l}(h)$ function for the investigated soils (solid lines) compared with numerical solutions of Eq. (7) (circles). 
Because the Tuller and Or (TO) model considers physical properties and processes, it is mathematically more complex than the empirical VG and $\mathrm{BC} K_{l}(h)$ relationships. The validity of Eq. (10) was evaluated using the TO model for Hygiene sandstone, Touchet silt loam, and Silt loam G.E.3 (the other soils were not included due to lack of availability of required information). For a detailed discussion interested readers are referred to Tuller and Or (2001).

The results presented in Fig. 6 indicate that the presented solution performs similarly well for the TO model despite the more complex physical and mathematical relationships between $K$ and $h$ considered in the TO model. These results thus may suggest that the new solution is generally independent of the relationship between $K$ and $h$ (whether simple or complex) and apparently works for all $K-h$ datasets for which $K$ decreases abruptly with increasing $h$ (i.e., coarse-textured media).

\subsubsection{The Durner (1994) bimodal $K_{l}(h)$ model}

Many natural soils and other porous media exhibit bimodal pore size distributions. These heterogeneous pore systems may be a result of specific particle size distributions or due to the formation of secondary pore systems by various soil genetic processes like aggregation, which results in two disparate populations of matrix and structural pores (Durner, 1994; Tuller and Or, 2002).

A soil with bimodal pore size distribution can be modeled with a linear superposition of VG curves (Durner, 1994). The unsaturated hydraulic conductivity function for a bimodal pore system composed of two van Genuchten type sub-curves is given as (Priesack and Durner, 2006):

$$
\begin{aligned}
K_{l}= & K_{s}\left[w S_{1}+(1-w) S_{2}\right]^{0.5} \\
& \times \frac{\left\{w \alpha_{1}\left[1-\left(1-S_{1}^{1 / m_{1}}\right)^{m_{1}}\right]+(1-w) \alpha_{2}\left[1-\left(1-S_{2}^{1 / m_{2}}\right)^{m_{2}}\right]\right\}^{2}}{\left[w \alpha_{1}+(1-w) \alpha_{2}\right]^{2}}
\end{aligned}
$$

where subscripts 1 and 2 corresponds to the first and second subcurves, $w$ is a weighting factor, and $S_{1}$ and $S_{2}$ are defined as:

$$
S_{1}=\left[1+\left(\alpha_{1} h\right)^{n_{1}}\right]^{-m_{1}}, \quad S_{2}=\left[1+\left(\alpha_{2} h\right)^{n_{2}}\right]^{-m_{2}}
$$

To test the validity of Eq. (10) for bimodal pore systems, the Durner $K(h)$ model was parameterized using a mixture of Sand 1 and Silt loam G.E.3 with varying fractions (varying $w$ ). The effective $K_{s}$ was assumed as the weighted average of $K_{s}$ for the two soils.

Numerical solutions of Eq. (7) for the bimodal $K_{l}(h)$ function (Eq. (19)) compared with the bimodal $K_{l}(h)$ function are depicted in Fig. 7. Because the $e-D_{\max }$ data pairs coincide with the $K(h)$ curve

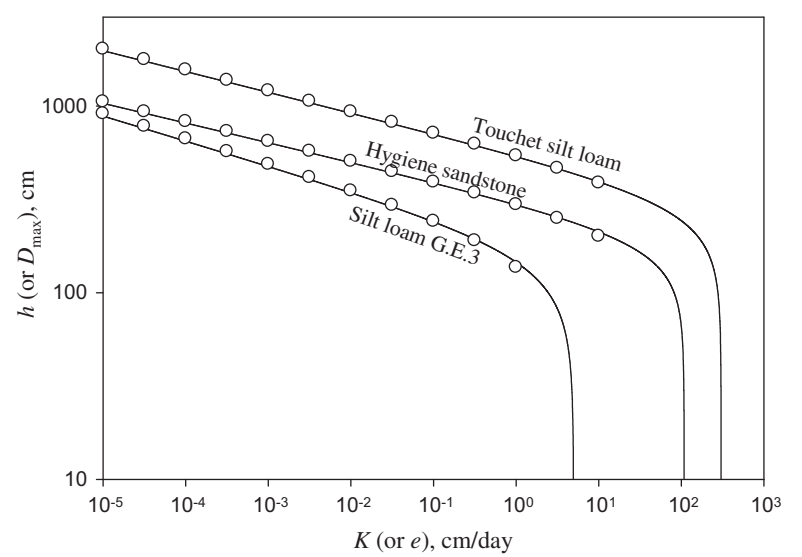

Fig. 6. The Tuller and Or $K_{l}(h)$ model for Hygiene sandstone, Touchet silt loam, and Silt loam G.E.3 (solid lines) compared with numerical solutions of Eq. (7) (circles). over most of the considered matric potential range, the proposed solution is generally valid for the bimodal $K_{l}(h)$ function as well. Estimation errors can be observed at the transition from the primary to the secondary sub-curves. In general, the bimodal $K_{l}(h)$ function exhibits a sharper transition than the numerical $e-D_{\max }$ data show. As indicated above, the presented solution is valid for coarse-textured soils with a steep $K_{l}(h)$ function only. However, in the transition zone $K$ changes very slowly with increasing $h$ and thus the soil in this zone locally mimics a fine-textured soil. Therefore, the solution does not work well near the transition zone, especially when sharp transitions are predicted for the $K_{l}(h)$ function.

\subsection{Comparison with experimental data}

Eq. (10) may be applied to determine the $K_{l}(h)$ function for coarse-textured porous media. This approach seems to be an attractive alternative, as $e$ and $D_{\max }$ are easier to measure in a steady-state evaporation experiment than $K_{l}$ and $h$. The evaporation rate may be accurately measured with a balance as the temporal change of mass of a constant head device (e.g., Mariotte bottle) used to establish a constant water level within the evaporation column. As $D_{\max }$ is a macroscopic length between water table and drying front, it may be measured by means of dye tracers (Shokri et al., 2009; Shokri and Salvucci, 2011) or image analysis (as demonstrated in Lehmann et al. (2008)). Beyond these simple methods, the drying front position can also be determined with advanced measurement technologies such as neutron radiography (Shokri et al., 2008) or heat pulse techniques (Sakai et al., 2011; Deol et al., 2012).

The applicability of Eq. (10) for determination of $K(h)$ was evaluated based on experimental data from Anat (1965), Willis (1960), Kumar (1999), Gardner and Fireman (1958), and Hassan and Ghaibeh (1977). While Anat (1965) contained all required data, $D_{\max }$ was not provided for all other considered datasets. As both theory (Gardner, 1958) and measurements (Deol et al., 2012; Assouline et al., 2013) suggest that the DF depth can be commonly much smaller than the WT depth, we assumed $D_{\max } \approx D$ (WT depth) for the experiments of Willis (1960), Kumar (1999), Gardner and Fireman (1958), and Hassan and Ghaibeh (1977).

To explore the validity of this assumption, the WT and DF depths were also simulated using Eqs. (6), (11) and (13) assuming the VG or bimodal conductivity function for describing $K_{l}(h)$ and Eq. (4) for $K_{v}(h)$ functions (surface temperature and relative humidity were assumed to be equal to $20^{\circ} \mathrm{C}$ and $50 \%$, respectively).

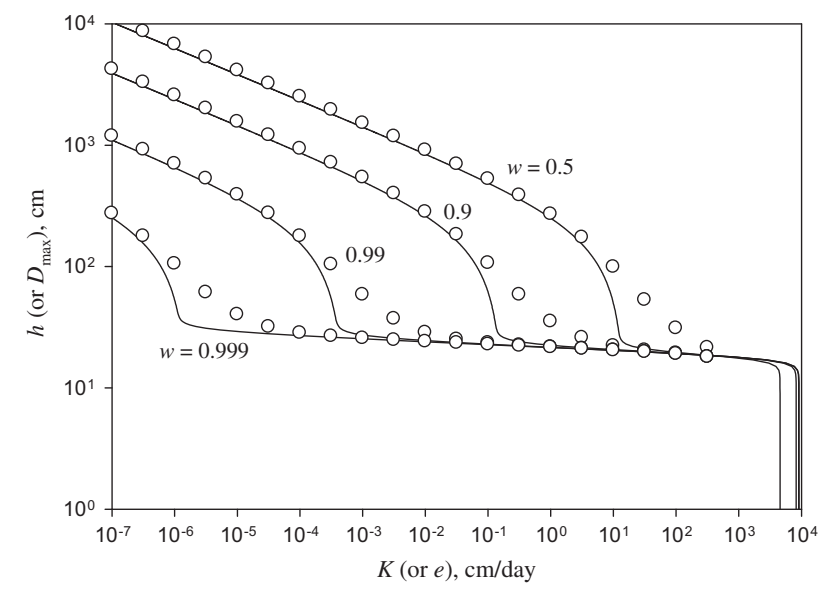

Fig. 7. Bimodal $K_{l}(h)$ function for $w$-weighted binary mixtures composed of Sand 1 and Silt loam G.E.3 (solid lines) compared with numerical solutions of Eq. (7) (circles). 
The $K_{l}(h)$ parameters were obtained by fitting Eq. (18) or (19) to the experimental $K(h)$ data and $\theta_{s}$ and $\theta_{r}$ were estimated/assumed based on soil water retention or textural data. It is worthwhile to note that the bimodal shape of the hydraulic conductivity data of some soils in this analysis could be due to film flow contribution rather than a heterogeneous pore system. Nonetheless, we found such soils can be adequately presented by the bimodal VG model (Eq. (19)) regardless of the physical causes for the bimodal shape.

Anat's (1965) data were collected from a steady-state upward liquid flow experiment holding the same governing flow equation as the steady-state evaporation process (within the liquid region) as implemented by Anat himself. In this experiment, the ambient atmospheric conditions were disregarded by inducing upward flow with an outflow siphon that was attached to a capillary barrier on top of the soil columns. Their experimental setup allowed establishment of very high rates (close to $K_{s}$ ) of steady upward flow from a water table.

The $D_{\max }$ defined as the distance between a nearly dry zone at the surface and the WT was measured and used in Anat's calculations. The dry zone at the soil surface was established by lowering the outflow siphon as far as possible to increase the pressure head at the surface up to a maximum $h$ at which liquid flow (obviously film flow) could still occur (i.e., just before hydraulic disruption occurs). This way Anat (1965) was able to increase surface $h$ up to about $1.5 h_{b}$, which likely lies just below $h_{\max }$ for such coarse soils (See $h_{\max }$ of Sand 1 in Fig. 4). We assumed that the dry zone in Anat's experiment has been the driest part of the partially saturated zone of Fig. 1 (just below the drying front), or in other words, we assumed Anat's measured $D_{\max }$ closely fits the theoretical definition considered here.

Fig. 8 presents the experimental data of Anat (1965), which overall indicates that $K-h$ and $e-D_{\max }$ data nearly coincide, especially for lower values of $e$ (steady upward flow rate in this case), while $e-D_{\max }$ data slightly underestimate $K-h$ data near saturation. The behavior of $e-D_{\max }$ data is similar to the solution of Eq. (7), which confirms the validity of the Buckingham-Darcy law for the process considered.

Experimental data of Willis (1960), Kumar (1999), Gardner and Fireman (1958), and Hassan and Ghaibeh (1977) are shown in Fig. 9. Reasonable agreement is shown between measured and simulated water table depths $(D)$ at various evaporation rates for most soils in Fig. 9 (except for the Chino clay), indicating the adequacy of the presented theory based on the Buckingham-Darcy law. Showing this law to be reasonably accurate for estimation of $D_{\max }$ (as shown in Fig. 8), we anticipate simulation results for the DF depth (i.e., $\delta=D-D_{\max }$ ) at different evaporation rates obtained by either numerical solution of Eq. (11) or its analytical solution,
Eq. (13), which are in excellent agreement with each other, to be adequate estimations for this analysis. These estimations then suggest that the DF depth remains within the top few $\mathrm{cm}$ of most soils for evaporation rates greater than $0.01 \mathrm{~cm} /$ day and $D$ can be considered nearly equal to $D_{\max }$ in this range, which further simplifies this approach as water table depth is even easier to measure than $D_{\text {max }}$. Note that these predictions were obtained assuming $T=20^{\circ} \mathrm{C}$ and $H_{r}=50 \%$ and might not be the case for significantly different environments especially when isothermal flow is not maintained.

The $e-D$ data are mostly in agreement with $K-h$ data except for the cases shown in Fig. 9e, f, and i in which pairs of $e-D$ lie above the $K-h$ data points. The disagreement in these cases likely stems from the very fine textures exhibited by these soils for which Eq. (10) does not hold as discussed above. Based on Fig. 9 results, which includes a broad range of soils (not only coarse-textured ones), we conclude that even for finer-textured soils, steady-state evaporation experiments can provide reasonable estimates leading to $K(h)$ relations.

\subsection{Estimation of effective unsaturated hydraulic conductivity of layered soils}

Based on the evaluation presented above we suggest that the steady-state evaporation rate can be used to infer unsaturated hydraulic conductivity of homogeneous coarse-textured soils. Since the steady-state evaporation rate is a macroscopic quantity, it can be considered as an effective value of unsaturated hydraulic conductivity for vertically stratified soil profiles. In other words, the resultant $K(h)$ values determined with Eq. (7) are representative for the entire $D_{\max }$ domain. This provides a unique opportunity for estimation of the effective unsaturated hydraulic conductivity of layered (stratified) soils that are quite common in natural ecosystems (Warrick, 2005).

To evaluate the validity of this concept the effective $K(h)$ curves for layered systems containing different soils with a constant water table at the bottom of the profile were considered. For this analysis, soil heterogeneity was described by Miller and Miller (1956) similar-media scaling theory. The VG conductivity function was assumed, Loveland sand was considered as a reference soil, and a set of similar soils were generated using the following relationship between VG parameters of each soil with those of the reference soil (Warrick et al., 1985):

$\alpha_{i}=\lambda \alpha_{R}, \quad K_{s i}=\lambda^{2} K_{s R}, \quad n_{i}=n_{R}$

where $\lambda$ is an arbitrary scaling factor, and subscripts $R$ and $i$ represent the reference soil and its similar soils, respectively.
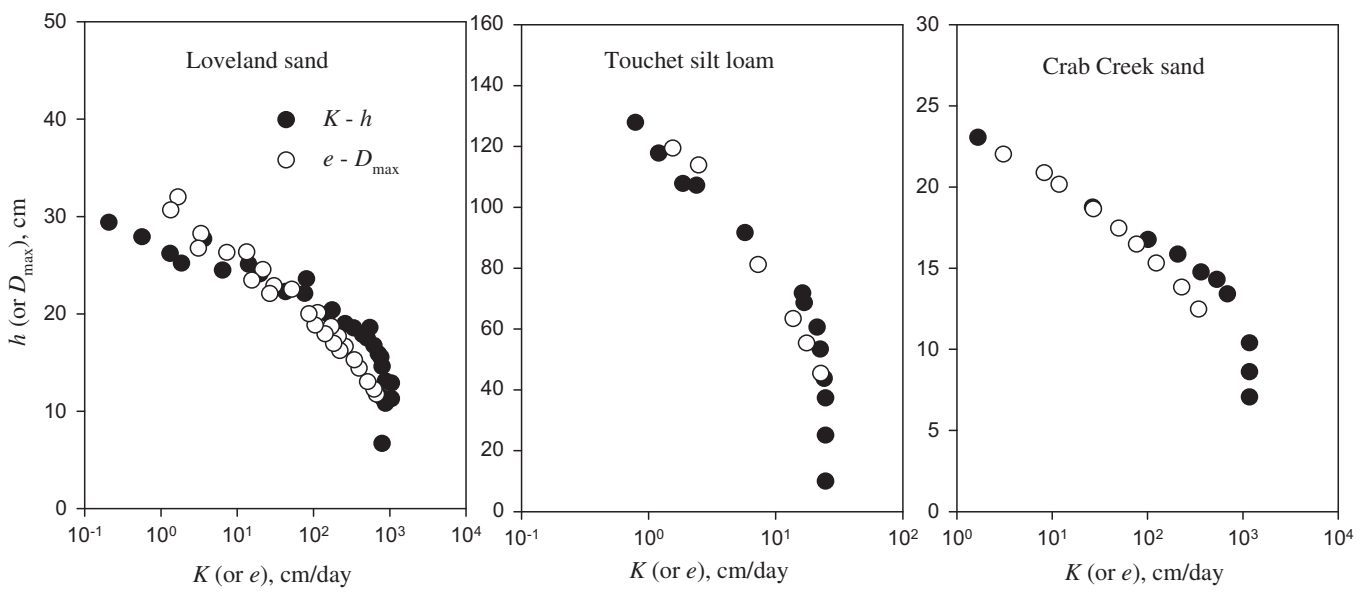

Fig. 8. Experimental data for unsaturated hydraulic conductivity and steady-state upward flow rate from Anat (1965). 

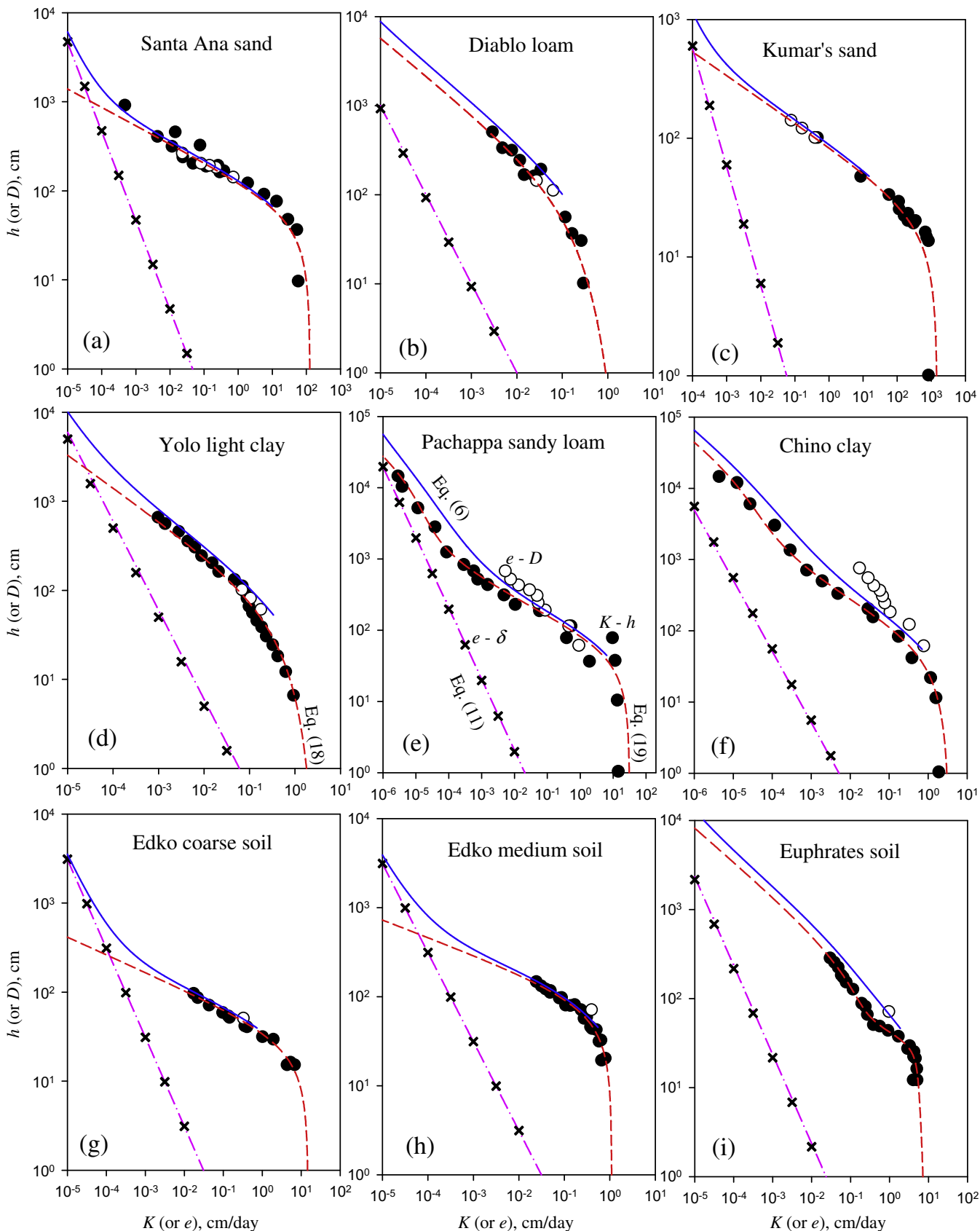

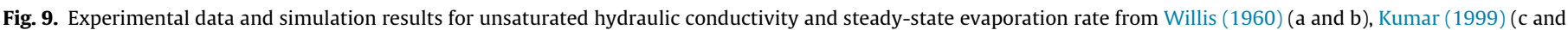

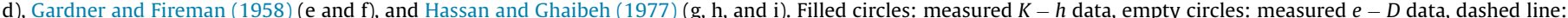

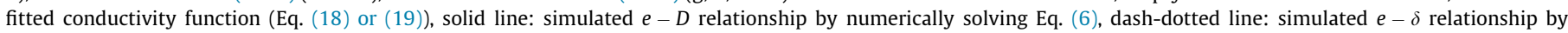
numerically solving Eq. (11), crosses: estimated $e-\delta$ relationship using Eq. (13). Note that $D_{\max }$ (not shown for clarity) is equal to $D-\delta \approx D$ in the range of $e-D$ data.

Fig. 10 presents results obtained for two 3-layer soil profiles, where the first has an increasing trend of $\lambda$ (i.e., getting finer) with depth and second has a decreasing trend of $\lambda$ (i.e., getting coarser) with depth. In Fig. 10a, the layered profile is composed of a $20-\mathrm{cm}$ layer of $\lambda=0.8$ at the top, $15-\mathrm{cm}$ of $\lambda=1$ in the middle, and $25-\mathrm{cm}$ of $\lambda=1.2$ at the bottom. The profile in Fig. $10 \mathrm{~b}$ consisted of $5-\mathrm{cm}$ of the $\lambda=1.2$ (top), $5-\mathrm{cm}$ of $\lambda=1$ (middle), and 35-cm of $\lambda=0.8$ (bottom). The thick solid line represents the $e-D_{\max }$ relationship obtained via numerical solution of Eq. (7). This curve can be considered equivalent to the effective hydraulic conductivity function of the layered profile (except near saturation). The effective $K(h)$ function was then applied for an equivalent homogeneous profile and Eq. (7) was solved again to yield a second set of $e-D_{\max }$ values depicted by circles in Fig. 10.
The agreement between the numerical solutions for the layered system and the introduced equivalent homogeneous profiles indicates the applicability of proposed approach for deriving effective (upscaled) hydraulic properties of layered soils. In Fig 10, a general relationship between the effective and the individual curves of the constituent soils can be observed. Each layer's individual curve contributes to the effective curve at $h$ values equal to the depth of that layer above the water table and the effective curve transitions from the individual curve of a bottom layer to individual curve of its top layer when $h$ exceeds the depth of the layers' interface to the water table.

Note that the domain for which the effective properties are determined is the unsaturated zone above the water table. As the hydraulic conductivity curve is the result of the status of the 

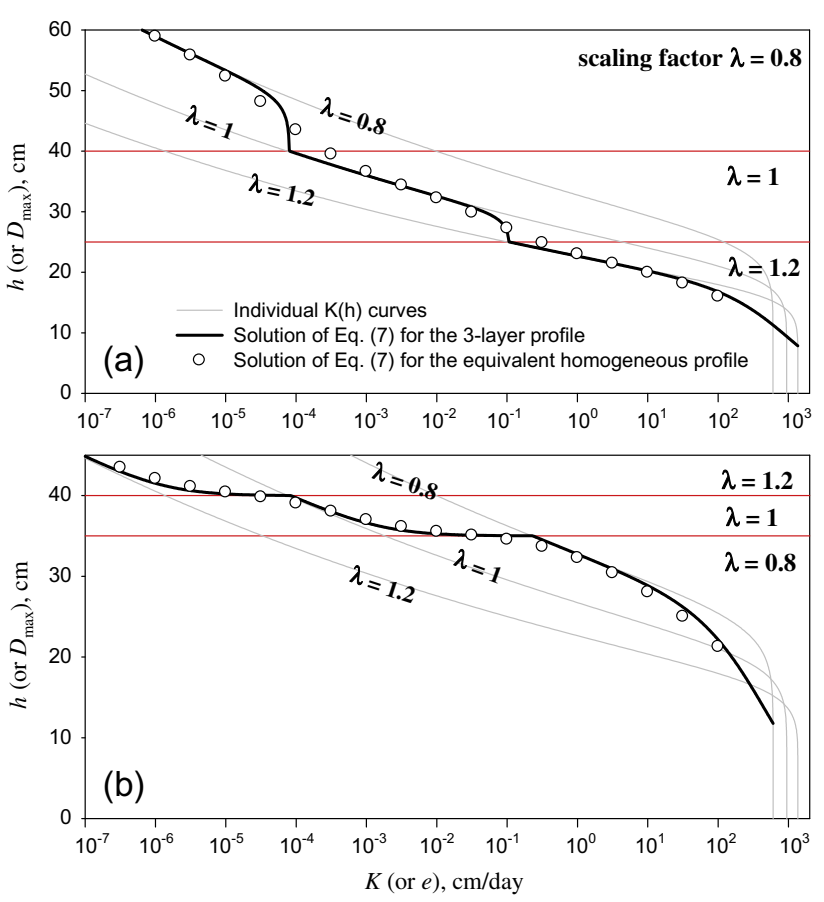

Fig. 10. Numerical solution of Eq. (7) for two 3-layer soil profiles (thick line) and for their equivalent homogenous soil profiles (circles). The former solution (thick line) was considered as the effective (upscaled) conductivity function of the equivalent homogenous profile. Horizontal lines show the layer interfaces.

system at different water tables, the domain for which the curve is determined is not constant but propagating into the soil profile depending on the extension of $D_{\max }$ above the water table.

It is quite well-known that a mulch layer (e.g. sand or gravel layer) at the soil surface can drastically reduce soil evaporation (Willis, 1960; Yuan et al., 2009; Shokri et al., 2010; Assouline et al., 2014). In order to study mulch effects with this new approach, a $40-\mathrm{cm}$ soil profile of $\lambda=0.8$ with a $3-\mathrm{cm}$ coarse layer $(\lambda=1.2)$ at the surface and a WT at the bottom was considered in Fig. 11. Consistent with findings in previous studies, this approach simply describes how a mulch layer reduces soil evaporation by adjusting the effective unsaturated hydraulic conductivity. For example, effective $K$ of the two-layer profile (coarse over fine) at $h=43 \mathrm{~cm}$ is about 4 orders of magnitude lower when compared to a homogeneous soil profile.

Effective hydraulic conductivity with the inverted profile layering (i.e., $3 \mathrm{~cm}$ of finer sand over $40 \mathrm{~cm}$ of coarser sand) is also presented in Fig. 11. In contrast to the coarse-over-fine layering which

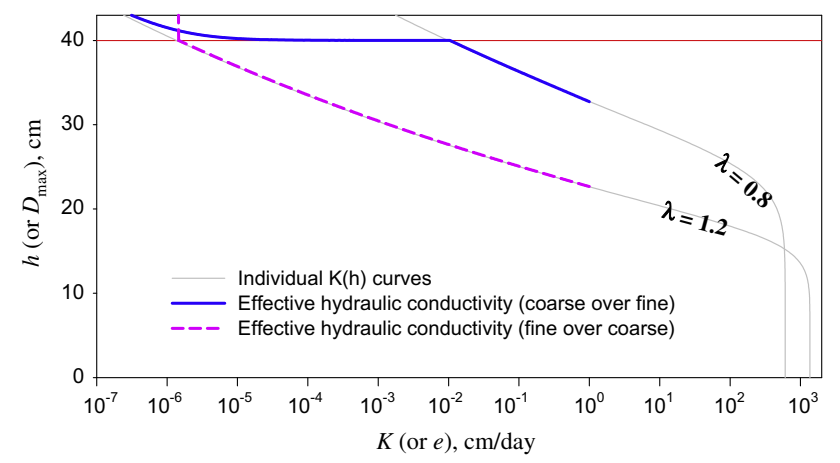

Fig. 11. Numerical solution of Eq. (7) (effective hydraulic conductivity) for a 40-cm homogeneous soil profile with a $3-\mathrm{cm}$ mulch layer at the surface and a water table at the bottom of the profile. shows very sharp transitions in the effective hydraulic conductivity curve, the transition in this case is slow and occurs in the range of very low conductivities. This behavior may provide a new description for the fact that the "difference in evaporation rate from a system of fine-textured soil overlying a coarse soil compared to a homogeneous profile of fine-textured soil may be relatively small, while the inverted layering condition may have a large effect on evaporation rates" (Willis, 1960).

This explanation is better understood considering Fig. 12. Learning from Fig. 10 that the effective conductivity for large pressure heads (larger than the profile depth, $D$ ) is the same as top layers' conductivity, the effective conductivity of the two layered profiles of Fig. $11(3 \mathrm{~cm}$ fine sand overlaying $40 \mathrm{~cm}$ coarse sand and $3 \mathrm{~cm}$ coarse sand overlaying $40 \mathrm{~cm}$ fine sand) for a broader range of pressure heads was calculated and plotted in Fig. 12 ( $K$ was considered as $K_{l}+K_{v}$ with $K_{v}$ calculated at $20^{\circ} \mathrm{C}$ ). This Figure clearly indicates a dramatic decrease in effective hydraulic conductivity of the coarse-over-fine profile compared to the homogeneous profile, while it shows no significant effect for the reverse layering.

In another scenario, we considered a $50-\mathrm{cm}$ multilayer profile composed of soils with $\lambda=1$ and $\lambda=0.5$ as sub-layers forming periodically repeated unit cells with various thicknesses; $5 \mathrm{~cm}$ (10 layers), $1 \mathrm{~cm}$ (50 layers), and $0.1 \mathrm{~cm}$ (500 layers). A constant water table was considered as the bottom boundary and numerical calculations were performed for layered and equivalent homogeneous profiles.

As shown in Fig. 13, solutions for the layered and for the equivalent homogeneous profiles are nearly identical. When thicker layers (i.e., 5-cm) are considered, the effective unsaturated conductivity curve (thick solid line) mimics that of a soil with a heterogeneous pore system similar to results presented in Figs. 10 and 11 . When the layer thicknesses are decreased, the effective curve approaches a homogeneous soil profile. This result is consistent with "homogenization theory" where a microscopically heterogeneous soil profile is represented as homogeneous at the macroscopic scale when the scale of heterogeneity is much smaller than the scale considered for modeling (e.g., Neuweiler and Eichel, 2006).

Fig. 13 also shows that the effective unsaturated hydraulic conductivity curve lies between the individual curves of the single layers and very closely matches the harmonic mean of the individual curves (except near saturation), which gets a stronger weighted contribution from smaller values. This result is similar to the well-known averaging method for saturated hydraulic conductivity of layered profiles that states that the effective hydraulic conductivity is the arithmetic or the harmonic mean of individual
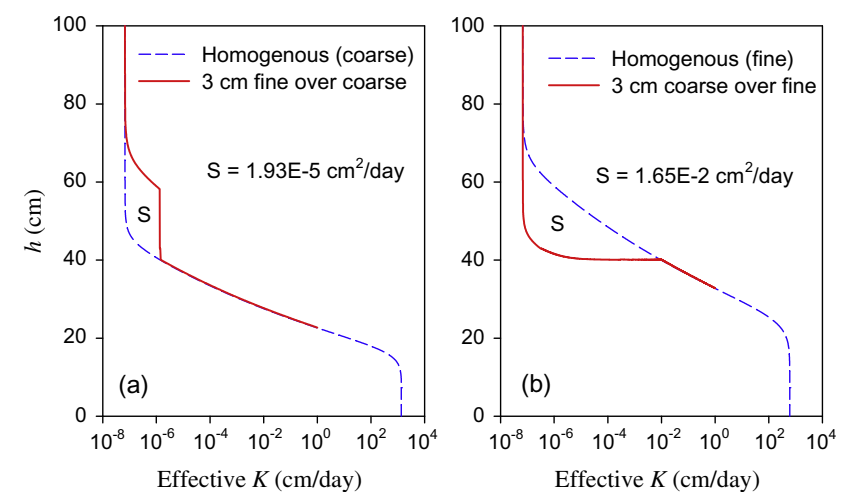

Fig. 12. Effective hydraulic conductivity for the layered soil profiles presented in Fig. 11 consisting of (a) $3 \mathrm{~cm}$ fine sand overlaying $40 \mathrm{~cm}$ coarse sand and (b) $3 \mathrm{~cm}$ coarse sand overlaying $40 \mathrm{~cm}$ fine sand. The area between homogeneous and layered conductivity curves $(S)$ is presented to show the difference between the two curves. 

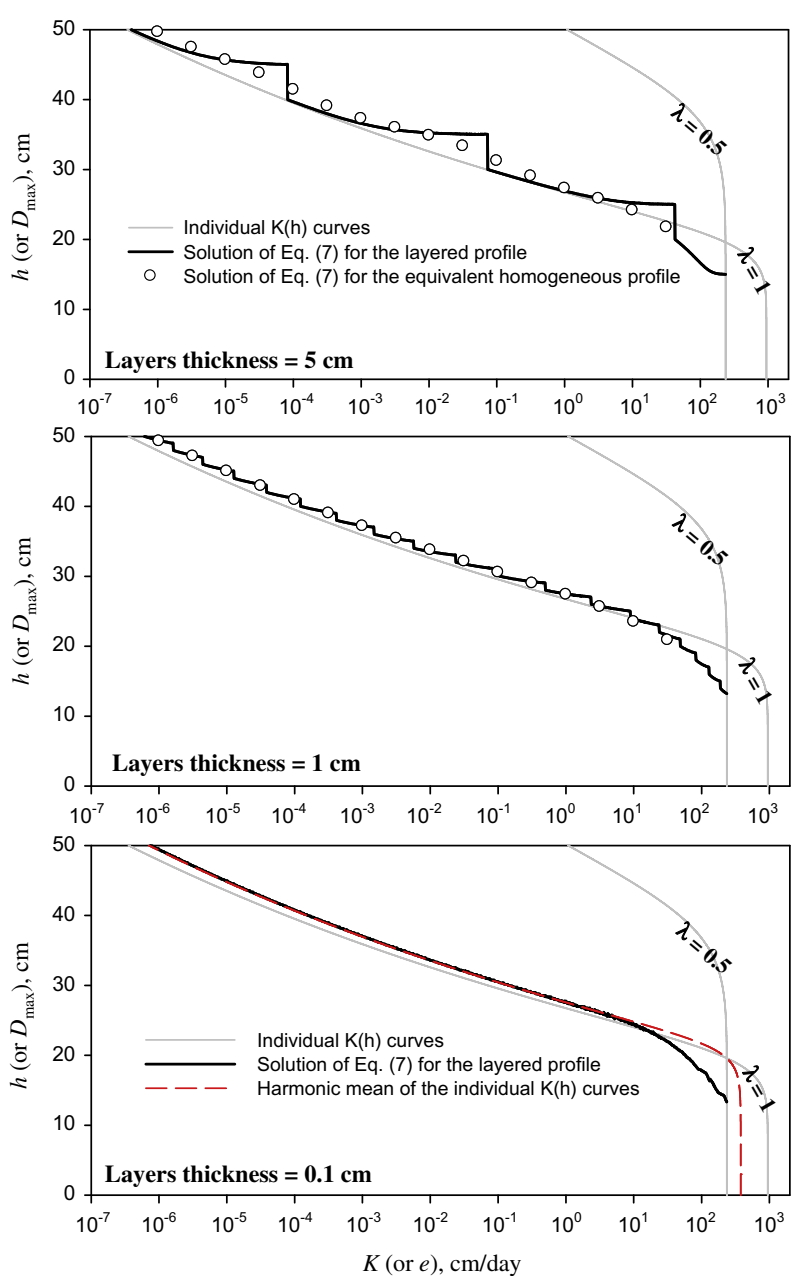

Fig. 13. Numerical solution of Eq. (7) for a $50-\mathrm{cm}$ multi-layer profile (thick line) composed of soils with $\lambda=1$ and $\lambda=0.5$ as sub-layers forming periodically repeated unit cells with various thicknesses and for its equivalent homogenous soil profile (circles). The former solution (thick line) was considered as the effective (upscaled) conductivity function of the equivalent homogenous profile.

layer conductivities for flow parallel or perpendicular to the layering, respectively (Freeze and Cherry, 1979).

The presented results are consistent with findings of other relevant studies of unsaturated hydraulic conductivity (e.g., Sarris and Paleologos, 2003; Warrick, 2005). Based on homogenization theory, Neuweiler and Eichel (2006) validated this result analytically for the same periodic heterogeneity structure with layer thicknesses much smaller than the profile depth. An advantage of the presented upscaling approach is that it is not restricted to binary heterogeneity structures, which is considered a limitation of homogenization theory. The presented method, however, is limited to a steady-state evaporation process.

\section{Summary and conclusions}

Based on evaluation of experimental and numerical data, the proposed new approach for steady-state evaporation (Eq. (10)) was shown to be valid for coarse-textured soils exhibiting a steep $K(h)$ function. Eq. (10) states that the steady-state evaporation rate from a constant water table is a macroscopic measure of unsaturated hydraulic conductivity in either homogeneous or layered coarse-textured media. Hence, in addition to a general approximate analytical solution for the Buckingham-Darcy law for $D_{\max }$, the presented approach offers a framework for determination of unsaturated hydraulic conductivity of homogeneous soils and more importantly a new solution for effective (upscaled) unsaturated hydraulic conductivity of layered soil systems.

Example applications of the proposed upscaling method for layered soils (Figs. 10-13) indicate its validity for defining effective hydraulic properties for a homogeneous soil equivalent to a given layered system. Hence, measuring pairs of $e-D_{\max }$ in a layered soil column during a steady-state evaporation experiment could provide a benchmark for evaluating the validity of existing theoretical approaches (e.g., inverse solutions, averaging schemes, homogenization theory) for upscaling hydraulic properties.

The domain for which the effective conductivity curve is determined by this method is not constant but propagating into the soil profile depending on the $D_{\max }$ extension above the water table. This is a direct consequence of the approach and should be taken into account when applying this method.

Only one-dimensional flow and thus one-dimensional heterogeneity (layering) was considered in this study. Application of the upscaling method to other heterogeneous media could be a subject of future studies. Both numerical modeling efforts and experimental studies on various other heterogeneous structures in two- or three-dimensional media are underway to expand the proposed approach.

\section{Acknowledgements}

The authors gratefully acknowledge support from the USDA NIFA AFRI Soil Processes Program under award 2009-6510705835 and by the Utah Agricultural Experiment Station, Utah State University, Logan, Utah 84322-4810, approved as journal paper no 8722.

\section{References}

Anat, A., 1965. Steady upward flow from water tables. Ph.D. dissertation. Colorado State University, Fort Collins, Colorado.

Assouline, S., Narkis, K., Gherabli, R., Lefort, P., Prat, M., 2014. Analysis of the impact of surface layer properties on evaporation from porous systems using column experiments and modified definition of characteristic length. Water Resour. Res. 50. http://dx.doi.org/10.1002/2013WR014489.

Assouline, S., Tyler, S.W., Selker, J.S., Lunati, I., Higgins, C.W., Parlange, M.B., 2013. Evaporation from a shallow water table: diurnal dynamics of water and heat at the surface of drying sand. Water Resour. Res. 49, 4022-4034.

Bechtold, M., Vanderborght, J., Weihermüller, L., Herbst, M., Günther, T., Ippisch, O. Kasteel, R., Vereecken, H., 2012. Upward transport in a three-dimensional heterogeneous laboratory soil under evaporation conditions. Vadose Zone J. http://dx.doi.org/10.2136/vzj2011.0066.

Bittelli, M., Ventura, F., Campbell, G.S., Snyder, R.L., Gallegati, F., Pisa, P.R., 2008 Coupling of heat, water vapor, and liquid water fluxes to compute evaporation in bare soils. J. Hydrol 362, 191-205.

Brooks, R.H., Corey, A.T., 1964. Hydraulic properties of porous media, Hydrol. Pap. 3, Colorado State Univ, Fort Collins, CO.

Buckingham, E., 1907. Studies on the movement of soil moisture. Bull., USDA, Bureau of Soils, vol. 38. Washington D.C.

Chen, Z, 2006. Recent advances of upscaling methods for the simulation of flow transport through heterogeneous porous media. J. Comput. Math. 24, 393-400.

Cushman, J.H., Bennethum, L.S., Hu, B.X., 2002. A primer on upscaling tools for porous media. Adv. Water Resour. 25, 1043-1067.

Deol, P., Heitman, J., Amoozegar, A., Ren, T., Horton, R., 2012. Quantifying nonisothermal sub-surface soil water evaporation. Water Resour. Res. 48, W11503.

Durner, W., 1994. Hydraulic conductivity estimation for soils with heterogeneous pore structure. Water Resour. Res. 30, 211-223.

Edelfsen, N.E., Anderson, A.B.C., 1943. The thermodynamics of soil moisture. Hilgardia 16, 31-298.

Erdal, D., Neuweiler, I., Huisman, J.A., 2012. Estimating effective model parameters for heterogeneous unsaturated flow using error models for bias correction. Water Resour. Res. 48, w06530.

Fayer, M.J., 2000. UNSAT-H version 3.0: unsaturated soil water and heat flow model. Theory, User Manual, and Examples. Pac. Northw. Natl. Lab., Richland, WA.

Freeze, R.A., Cherry, J.A., 1979. Groundwater. Prentice Hall, Englewood Cliffs, NJ.

Gardner, W.R., 1958. Some steady state solutions of the unsaturated moisture flow equation with application to evaporation from a water table. Soil Sci. 85, 228232.

Gardner, W.R., Fireman, M., 1958. Laboratory studies of evaporation from soil columns in the presence of a water table. Soil Sci. 85, 244-249. 
Gowing, J.W., Konukcu, F., Rose, D.A., 2006. Evaporative flux from a shallow watertable: the influence of a vapour-liquid phase transition. J. Hydrol. 321, 77-89.

Hassan, F.A., Ghaibeh, A.Sh., 1977. Evaporation and salt movement in soils in the presence of water table. Soil Sci. Soc. Am. J. 41, 470-478.

Hassanizadeh, S.M., Gray, W.G., 1979. General conservation equations for multiphase systems. 1. Averaging procedure. Adv. Water Resour. 2, 131-144.

Hassanizadeh, S.M., Gray, W.G., 1979. General conservation equations for multiphase systems. 2. Mass, momenta, energy, and entropy equations. Adv. Water Resour. 2, 191-208.

Hassanizadeh, S.M., Gray, W.G., 1980. General conservation equations for multiphase systems. 3. Constitutive theory for porous media. Adv. Water Resour. 3, 25-40.

Hopmans, J.W., Schukkinga, H., Torfs, P.J.J.F., 1998. Two-dimensional steady state unsaturated water flow in heterogeneous soils with autocorrelated soil hydraulic properties. Water Resour. Res. 24, 2005-2017.

Hopmans, J.W., Simunek, J., 1999. Review of inverse estimation of soil hydraulic properties. In: van Genuchten, M.Th., Leij, F.J., Wu, L. (Eds.), Characterization and Measurement of the Hydraulic Properties of Unsaturated Porous Media. University of California, Riverside, CA, pp. 643-659.

Hunt, A.G., 1998. Upscaling in subsurface transport using cluster statistics of percolation. Transp. Porous Media 30, 177-198.

Hunt, A.G., Idriss, B., 2009. Percolation-based effective conductivity calculations for bimodal distributions of local conductances. Phil. Mag. 89, 22-24.

Javaux, M., Vanclooster, M., 2006. Scale-dependency of the hydraulic properties of a variably saturated heterogeneous sandy subsoil. J. Hydrol. 327, 376-388.

King, P.R., 1989. The use of renormalization for calculating effective permeability. Transp. Porous Media 4, 37-58.

King, P.R., Neuweiler, I., 2002. Probability upscaling. Comput. Geosci. 6, 101-114.

Kitanidis, P.K., 1990. Effective hydraulic conductivity for gradually varying flow. Water Resour. Res. 26, 1197-1208.

Kosugi, K., 1996. Lognormal distribution model for unsaturated soil hydraulic properties. Water Resour. Res. 32, 2697-2703.

Kosugi, K., Hopmans, J.W., 1998. Scaling water retention curves for soils with lognormal pore-size distribution. Soil Sci. Soc. Am. J. 62, 1496-1504.

Kumar, C.P., 1999. Evaporation from shallow water table through layered soil profiles. ISH J. Hydraulic Eng. 5, 65-75.

Lehmann, P., Assouline, S., Or, D., 2008. Characteristic lengths affecting evaporative drying of porous media. Phys. Rev. E 77, 56309.

Lu, Z., Zhang, D., 2004. Analytical solutions to steady state unsaturated flow in layered, randomly heterogeneous soils via Kirchhoff transformation. Adv. Water Resour. 27, 775-784.

Neuweiler, I., Cirpka, O.A., 2005. Homogenization of Richards equation in permeability fields with different connectivities. Water Resour. Res. 41, W02009.

Neuweiler, I., Eichel, H., 2006. Effective parameter functions for Richards equation in layered porous media. Vadose Zone J. 5, 963-977.

Neuweiler, I., Vogel, H.J., 2007. Upscaling for unsaturated flow for non-Gaussian heterogeneous porous media. Water Resour. Res. 43, w03443.

Miller, E.E., Miller, R.D., 1956. Physical theory for capillary flow phenomena. J. Appl. Phys. 27, 324-332.

Millington, R.J., Quirk, J.P., 1961. Permeability of porous media. Trans. Faraday Soc. 57, 1200-1207.

Moore, R.E., 1939. Water conduction from shallow water tables. Hilgardia 12, $383-$ 426.

Mualem, Y., 1976. A new model for predicting the hydraulic conductivity of unsaturated porous media. Water Resour. Res. 12, 513-521.

Ojha, R., Morbidelli, R., Saltalippi, C., Flammini, A., Govindaraju, R.S., 2014. Scaling of surface soil moisture over heterogeneous fields subjected to a single rainfall event. J. Hydrol. 516, 21-36.

Or, D., Lehmann, P., Shahraeeni, E., Shokri, N., 2013. Advances in soil evaporation physics: a review. Vadose Zone J. 12, 1-16.

Or, D., Tuller, M., 1999. Liquid retention and interfacial area in variably saturated porous media: upscaling from single pore to sample scale model. Water Resour. Res. 35 (12), 3591-3606.

Peters, A., 2013. Simple consistent models for water retention and hydraulic conductivity in the complete moisture range. Water Resour. Res. 49, 6765-6780.

Peters, A., Durner, W., 2010. Reply to comment by N. Shokri and D. Or on "A simple model for describing hydraulic conductivity in unsaturated porous media accounting for film and capillary flow". Water Resour. Res. 46, W06802.

Philip, J.R., de Vries, D.A., 1957. Moisture movement in porous materials under temperature gradient. Trans. Am. Geophys. Union 38, 222-232.

Prevedello, C.L., Loyola, J.M.Th., Reichardt, K., Nielsen, D.R., 2009. New analytic solution related to the Richards, Philip, and Green-ampt equations for infiltration. Vadose Zone J. 8, 127-135.

Priesack, E., Durner, W., 2006. Closed-form expression for the multimodal unsaturated conductivity function. Vadose Zone J. 5, 121-124.

Pachepsky, Y., Radcliffe, D., Selim, H.M., 2003. Scaling Methods in Soil Physics. CRC Press, Boca Raton, FL.

Ripple, C.D., Rubin, J., van Hylckama, T.E.A., 1970. Estimating steady-state evaporation rates from bare soils under conditions of high water table. Water-Supply Paper 2019-A. US Geological Survey, Washington, DC.

Sadeghi, M., Shokri, N., Jones, S.B., 2012a. A novel analytical solution to steady-state evaporation from porous media. Water Resour. Res. 48, W09516.

Sadeghi, M., Ghahraman, B., Ziaei, A.N., Davary, K., Reichardt, K., 2012b. Invariant solutions of Richards' equation for water movement in dissimilar soils. Soil Sci. Soc. Am. J. 76, 1-9.
Sadeghi, M., Ghahraman, B., Ziaei, A.N., Davary, K., Reichardt, K., 2012c. Additional scaled solutions to Richards' equation for infiltration and drainage. Soil Till. Res. 119, 60-69.

Sadeghi, M., Jones, S.B., 2012. Scaled solutions to coupled soil-water flow and solute transport during the redistribution process. Vadose Zone J. 11 (4). http:// dx.doi.org/10.2136/vzj2012.0023.

Sakai, M., Jones, S.B., Tuller, M., 2011. Numerical evaluation of subsurface soil water evaporation derived from sensible heat balance. Water Resour. Res. 47, W02547.

Salvucci, G.D., 1993. An approximate solution for steady vertical flux of moisture through an unsaturated homogeneous soil. Water Resour. Res. 29 (11), 37493753.

Samouelian, A., Vogel, H.J., Ippisch, O., 2007. Upscaling hydraulic conductivity based on the topology of the sub-scale structure. Adv. Water Resour. 30, 1179-1189.

Sarris, T.S., Paleologos, E.K., 2003. Numerical investigation of the anisotropic hydraulic conductivity behavior in heterogeneous porous media. Stoch. Environ. Res. 18, 1-10.

Saucier, A., 1992. Effective permeability of multifractal porous media. Physica A 183, 381-397.

Shokri, N., Lehmann, P., Vontobel, P., Or, D., 2008. Drying front and water content dynamics during evaporation from sand delineated by neutron radiography. Water Resour. Res. 44, W06418.

Shokri, N., Lehmann, P., Or, D., 2009. Critical evaluation of enhancement factors for vapor transport through unsaturated porous media. Water Resour. Res. 45 W10433. http://dx.doi.org/10.1029/2009WR007769.

Shokri, N., Or, D., 2010. Comment on "A simple model for describing hydraulic conductivity in unsaturated porous media accounting for film and capillary flow" by A. Peters and W. Durner. Water Resour. Res. 46, W06801. http:// dx.doi.org/10.1029/2009WR008917.

Shokri, N., Lehmann, P., Or, D., 2010. Evaporation from layered porous media. J. Geophys. Res. 115, B06204.

Shokri, N., Salvucci, G.D., 2011. Evaporation from porous media in the presence of a water table. Vadose Zone J. 10, 1309-1318.

Smits, K.M., Ngo, V.V., Cihan, A., Sakaki, T., Illangasekare, T.H., 2012. An evaluation of models of bare soil evaporation formulated with different land surface boundary conditions and assumptions. Water Resour. Res. 48, W12526.

Tuller, M., Or, D., Dudley, L.M., 1999. Adsorption and capillary condensation in porous media - liquid retention and interfacial configurations in angular pores. Water Resour. Res. 35 (7), 1949-1964.

Tuller, M., Or, D., 2001. Hydraulic conductivity of variably saturated porous media: film and corner flow in angular pore space. Water Resour. Res. 37, 1257-1276.

Tuller, M., Or, D., 2002. Unsaturated hydraulic conductivity of structured porous media: a review of liquid configuration-based models. Vadose Zone J. 1, 14-37.

van Dam, J.C., Stricker, J.N.M., Droogers, P., 1994. Inverse method to determine soil hydraulic functions from multistep outflow experiments. Soil. Sci. Am. J. 58 647-652.

van Genuchten, M.T., 1980. A closed-form equation for predicting the hydraulic conductivity of unsaturated soils. Soil Sci. Soc. Am. J. 44, 892-898.

Vereecken, H., Kasteel, R., Vanderborght, J., Harter, T., 2007. Upscaling hydraulic properties and soil water flow processes in heterogeneous soils: a review. Vadose Zone J. 6, 1-28.

Vrugt, J.A., Stauffer, P.H., Wöhling, Th., Robinson, B.A., Vesselinov, V.V., 2008. Inverse modeling of subsurface flow and transport properties: a review with new developments. Vadose Zone J. 7, 843-864.

Warrick, A.W., 2005. Effective unsaturated hydraulic conductivity for onedimensional structured heterogeneity. Water Resour. Res. 41, w09406.

Warrick, A.W., 1988. Additional solutions for steady-state evaporation from a shallow water table. Soil Sci. 146, 63-66.

Warrick, A.W., Amoozegar-Fard, A., 1979. Infiltration and drainage calculations using spatially scaled hydraulic properties. Water Resour. Res. 15, 1116-1120.

Warrick, A.W., Lomen, D.O., Yates, S.R., 1985. A generalized solution to infiltration. Soil Sci. Soc. Am. J. 49, 34-38.

Warrick, A.W., Yeh, T.C.J., 1990. One-dimensional, steady vertical flow in a layered soil profile. Adv. Water Resour. 13, 207-210.

Wen, X.H., Gomez-Hernandez, J.J., 1996. Upscaling hydraulic conductivities in heterogeneous media: an overview. J. Hydrol. 183, ix-xxxii.

Willis, W.O., 1960. Evaporation from layered soils in the presence of a water table. Soil Sci. Soc. Am. Proc. 24, 239-242.

Yeh, T.C.J., Gelhar, L.W., Gutjahr, A.L., 1985. Stochastic analysis of unsaturated flow in heterogenous soils. 1.Statistically isotropic media. Water Resour. Res. 21,447-456.

Yeh, T.C.J., Harvey, D., 1990. Effective unsaturated hydraulic conductivity of layered sands. Water Resour. Res. 26, 1271-1279.

Yuan, C., Lei, T., Mao, L., Liu, H., Wu, Y., 2009. Soil surface evaporation processes under mulches of different sized gravel. Catena 78, 117-121.

Zhang, D., Lu, Z., 2002. Stochastic analysis of flow in a heterogeneous unsaturatedsaturated system. Water Resour. Res. 38, 1018.

Zhang, Z.F., Ward, A.L., Gee, G.W., 2004. A combined parameter scaling and inverse technique to upscale the unsaturated hydraulic parameters for heterogeneous soils. Water Resour. Res. 40, w08306.

Zhu, J., Mohanty, B.P., 2002. Spatial averaging of van Genuchten hydraulic parameters for steady-state flow in heterogeneous soils: a numerical study. Vadose Zone J. 1, 261-272, <www.vadosezonejournal.org>.

Zhu, J., Mohanty, B.P., Warrick, A.W., van Genuchten, M.Th., 2004. Correspondence and upscaling of hydraulic functions for steady state flow in heterogeneous soils. Vadose Zone J. 3, 527-533, <www.vadosezonejournal.org>.

Zhu, J., Mohanty, B.P., 2006. Effective scaling factor for transient infiltration in heterogeneous soils. J. Hydrol. 319, 96-108. 